\title{
AN EXTENSION OF DARBO'S THEOREM AND ITS APPLICATION TO SYSTEM OF NEUTRAL DIFFERENTIAL EQUATIONS WITH DEVIATING ARGUMENT
}

\author{
SH. BANAEI, M. B. GHAEMI, AND R. SAADATI \\ Received 12 September, 2016
}

\begin{abstract}
In this paper we extend Darbo's fixed point theorem in Banach spaces and obtain a tripled fixed point theorem. The technique of measure of noncompactness is the main tool in carrying out our proof. Finally, as an application of our results, we analyze the existence of solutions for a system of neutral differential equations.
\end{abstract}

2010 Mathematics Subject Classification: 47H08; 47H10

Keywords: measure of noncompactness, fixed point theorem, tripled fixed point theorem, integral equation

\section{INTRODUCTION AND PRELIMINARIES}

The notion of a measure of non compactness (MNC) was introduced by Kuratowski [9] in 1930. Darbo's fixed point theorem [7] which ensures the existence of fixed point is an important application of this measure, since it generalizes both Schauder fixed point and Banach contraction principle. Thereafter, many investigations and papers have been appeared in the MNC and it's application, see for example $[1,2,5,8,10]$. The aim of this paper is to generalize the Darbo's fixed point theorem via the concept of the class of operators $O(f ;$.$) and extend the results of the theorem$ presented by Samadi and Ghaemi in [10]. Further, we apply our extension to obtain a tripled fixed point and study the existence of solutions for the system

$$
\left\{\begin{array}{l}
x^{\prime}(t)=f\left(t, x(\zeta(t)), y(\zeta(t)), z(\zeta(t)), x^{\prime}(\beta(t)), y^{\prime}(\beta(t)), z^{\prime}(\beta(t))\right) \\
y^{\prime}(t)=f\left(t, y(\zeta(t)), x(\zeta(t)), z(\zeta(t)), y^{\prime}(\beta(t)), x^{\prime}(\beta(t)), z^{\prime}(\beta(t))\right) \\
z^{\prime}(t)=f\left(t, z(\zeta(t)), y(\zeta(t)), x(\zeta(t)), z^{\prime}(\beta(t)), y^{\prime}(\beta(t)), x^{\prime}(\beta(t))\right)
\end{array}\right.
$$

where $t \in[0, T]$ with the initial condition

$$
x(0)=x_{0}, y(0)=y_{0}, z(0)=z_{0} .
$$


First, we introduce some notations and definitions which are used throughout this paper. Let $R$ be the set of real numbers, $R_{+}=[0, \infty)$ and $(E,\|\|$.$) be a real Banach$ space with the zero element 0 . We write $\bar{B}(x, r)$ to denote the closed ball centered at $x$ with radius $r$. If $X$ be a nonempty subset of $E$ then the symbols $\bar{X}$ and $\operatorname{Conv} X$ stand for the closure and closed convex hull of $X$, respectively. Moreover, $\mathfrak{M}_{E}$ is the family of nonempty bounded subset of $E$ and $\mathfrak{N}_{E}$ denote its subfamily consisting of all relatively compact sets.

Definition 1 ([6]). A mapping $\mu: \mathfrak{M}_{E} \longrightarrow R_{+}$is said to be a measure of noncompactness in $\mathrm{E}$ if it satisfies the following conditions:

$1^{\circ}$ The family ker $\mu=\left\{X \in \mathfrak{M}_{E}: \mu(X)=0\right\}$ is nonempty and ker $\mu \subset \mathfrak{N}_{E}$;

$2^{\circ} X \subset Y \Longrightarrow \mu(X) \leq \mu(Y)$;

$3^{\circ} \mu(\bar{X})=\mu(X)$;

$4^{\circ} \mu(\operatorname{Conv} X)=\mu(X)$;

$5^{\circ} \mu(\lambda X+(1-\lambda) Y) \leq \lambda \mu(X)+(1-\lambda) \mu(Y)$ for $\lambda \in[0,1]$;

$6^{\circ}$ If $\left\{X_{n}\right\}$ is a sequence of closed sets from $\mathfrak{M}_{E}$ such that $X_{n+1} \subset X_{n}$ for $n=1,2, \cdots$, and if $\lim _{n \rightarrow \infty} \mu\left(X_{n}\right)=0$, then $X_{\infty}=\cap_{n=1}^{\infty} X_{n} \neq \varnothing$.

The following concept of $O(f ;$.$) was given by Altun and Turkoglu [4].$

Let $F([0, \infty))$ be the class of all functions $f:[0, \infty) \rightarrow[0, \infty)$ and let $\Theta$ be the class of all operators

$O(\bullet ; \cdot): F([0, \infty)) \rightarrow F([0, \infty)), \quad f \rightarrow O(f ; \cdot)$

satisfying the following conditions:

(1) $O(f ; t)>0$ for $t>0$ and $O(f ; 0)=0$.

(2) $O(f ; t) \leq O(f ; s)$ for $t \leq s$.

(3) $\lim _{n \rightarrow \infty} O\left(f ; t_{n}\right)=O\left(f ; \lim _{n \rightarrow \infty} t_{n}\right)$.

(4) $O(f ; \max \{t, s\})=\max \{O(f ; t), O(f ; s)\}$ for some $f \in F([0, \infty))$.

Example 1. ([4] ) If $f:[0, \infty) \rightarrow[0, \infty)$ is a nondecreasing, continuous function such that $f(0)=0$ and $f(t)>0$ for $t>0$, then the operator defined by

$$
O(f ; t)=\frac{f(t)}{1+f(t)}
$$

satisfies the above conditions.

Definition 2 ([8]). A triple $(x, y, z)$ is called a triple fixed point of a mapping $T$ : $X \times X \times X \rightarrow X$ if

$$
T(x, y, z)=x, \quad T(y, x, z)=y, \quad T(z, y, x)=z .
$$

The following theorems are basic for our main results.

Theorem 1 (Schauder [3]). Let $C$ be a nonempty, bounded, closed and convex subset of a Banach space $E$. Then every compact and continuous map $T: C \rightarrow C$ has at least one fixed point. 
Theorem 2 (Darbo [7]). Let $C$ be a nonempty, bounded, closed and convex subset of a Banach space $E$ and $T: C \rightarrow C$ be a continuous mapping. Assume that there exists a constant $K \in[0,1)$ such that $\mu(T X) \leq K \mu(X)$ for any nonempty subset $X$ of $C$, where $\mu$ is a MNC defined in $E$. Then $T$ has at least a fixed point in $C$.

Theorem 3 ([10]). Suppose $\mu_{1}, \mu_{2}, \ldots, \mu_{n}$ are measures of noncompactness in Banach spaces $E_{1}, E_{2}, \ldots, E_{n}$ respectively. Moreover assume that the function $F$ : $R_{+}^{n} \longrightarrow R_{+}$is convex and $F\left(x_{1}, \ldots, x_{n}\right)=0$ if and only if $x_{i}=0$ for $i=1,2, \ldots, n$. Then

$$
\tilde{\mu}(X)=F\left(\mu_{1}\left(X_{1}\right), \mu_{2}\left(X_{2}\right), \ldots, \mu_{n}\left(X_{n}\right)\right),
$$

defines a measure of noncompactness in $E_{1} \times E_{2} \times \ldots \times E_{n}$ where $X_{i}$ denotes the natural projection of $X$ into $E_{i}$, for $i=1,2, \ldots, n$.

Remark 1. Aghajani et al. [1] illustrated the Theorem 3 by the following example. Let $F$ be as follows:

$$
F(x, y, z)=\max \{x, y, z\} \text {, or } F(x, y, z)=x+y+z \text {, for any }(x, y, z) \in[0, \infty)^{3} \text {. }
$$

They showed that

$\widetilde{\mu}(X)=\max \left(\mu_{1}\left(X_{1}\right), \mu_{2}\left(X_{2}\right), \mu_{3}\left(X_{3}\right)\right)$, or $\widetilde{\mu}(X)=\mu_{1}\left(X_{1}\right)+\mu_{2}\left(X_{2}\right)+\mu_{3}\left(X_{3}\right)$, defines an MNC in the space $E_{1} \times E_{2} \times E_{3}$ where $X_{i},(i=1,2,3)$ are the natural projection of $X$ into $E_{i}$.

Samadi and Ghaemi [10] generalized Darbo's fixed point theorem as fallow.

Theorem 4. Let $U$ be a nonempty, bounded, closed and convex subset of a Banach space E. Assume $F: U \rightarrow U$ be a continuous operator such that satisfying

$$
\psi(\mu(F(X))) \leq \phi(\psi(\mu(X))) \psi(\mu(X))
$$

for all nonempty subset $X$ of $U$, where $\mu$ is an arbitrary $M N C$ in $E, \psi: R_{+} \rightarrow R_{+}$is nondecreasing function such that $\psi(t)=0$ if and only if $t=0$ and $\phi:[0, \infty) \rightarrow[0,1)$ is a continuous function such that $\limsup _{t \rightarrow r} \phi(t)<1$ for all $r \geq 0$. Then $F$ has a fixed point in $U$.

The Darbo's fixed point theorem is followed if $\psi=I$ (identity map) and $\phi=K$ in the Theorem 4.

\section{MAIN RESULTS}

Now we state one of the main results in this paper which extends and generalizes Darbo's fixed point theorem by using concept of $O(f ;$.$) .$

Theorem 5. Let $C$ be a nonempty, bounded, closed and convex subset of a Banach space $E$ and $T: C \rightarrow C$ be a continuous operator such that

$$
\psi[O(f ; \mu(T X))] \leq \Phi[\psi(O(f ; \mu(X)))] \psi(\mu(X)),
$$


for $X$ of $C, O(\bullet ; \cdot) \in \Theta$ and let $\psi: R_{+} \rightarrow R_{+}$be a nondecreasing function such that $\psi(t)=0$ if and only if $t=0$. Let $\Phi:[0, \infty) \rightarrow[0,1)$ is a continuous function such that $\lim _{n \rightarrow \infty} \Phi^{n}(t)=0$ for each $t \geq 0$, where $\mu$ is an arbitrary $M N C$. Then $T$ has at least one fixed point in $C$.

Proof. By induction, we construct a sequence $\left\{C_{n}\right\}$ such that $C_{0}=C$ and $C_{n+1}=$ $\operatorname{Conv}\left(T C_{n}\right)$ for $n \geq 1$. We have

$T C_{0}=T C \subseteq C=C_{0}, C_{1}=\operatorname{Conv}\left(T C_{0}\right) \subseteq C=C_{0}$, therefore by continuing this process deduce that

$$
C_{0} \supseteq C_{1} \supseteq \ldots \supseteq C_{n} \supseteq C_{n+1} \supseteq \ldots
$$

If there exists an integer $N \geq 0$ such that $\mu\left(C_{N}\right)=0$, then $C_{N}$ is relatively compact. In this case Theorem 1 implies that $T$ has a fixed point. Now we suppose that $\mu\left(C_{n}\right) \neq 0$ for $n \geq 0$. We have

$$
\begin{aligned}
\psi\left[O\left(f ; \mu\left(C_{n+1}\right)\right)\right] & =\psi\left[O\left(f ; \mu\left(\operatorname{Conv}\left(T C_{n}\right)\right)\right)\right] \\
& =\psi\left[O\left(f ; \mu\left(T C_{n}\right)\right)\right] \\
& \leq \Phi\left[\psi\left(O\left(f ; \mu\left(C_{n}\right)\right)\right)\right] \psi\left(\mu\left(C_{n}\right)\right) \\
& \leq \Phi^{2}\left[\psi\left(O\left(f ; \mu\left(C_{n-1}\right)\right)\right)\right] \psi\left(\mu\left(C_{n-1}\right)\right) \\
& \vdots \\
& \leq \Phi^{n}\left[\psi\left(O\left(f ; \mu\left(C_{0}\right)\right)\right)\right] \psi\left(\mu\left(C_{0}\right)\right) \\
& =\Phi^{n}[\psi(O(f ; \mu(C))] \psi(\mu(C))
\end{aligned}
$$

By letting $n \rightarrow \infty$ in (1.2) we infer that $\lim _{n \rightarrow \infty} \psi\left[O\left(f ; \mu\left(C_{n+1}\right)\right)\right]=0$. Therefore,

$$
\lim _{n \rightarrow \infty} \psi\left[O\left(f ; \mu\left(C_{n+1}\right)\right)\right]=\psi\left[O\left(f ; \lim _{n \rightarrow \infty} \mu\left(C_{n+1}\right)\right)\right]=0,
$$

we know that $O(f ; 0)=0$. Thus,

$\lim _{n \rightarrow \infty} \mu\left(C_{n+1}\right)=0$. Since $C_{n} \supseteq C_{n+1}$ and $T C_{n} \subseteq C_{n}$ for all $n=1,2,3, \ldots$ then from $6^{\circ}$ of definition MNC, $X_{\infty}=\cap_{n=1}^{\infty} X_{n}$ is a nonempty, closed and convex set, invariant under $T$ and belongs to $\operatorname{Ker} \mu$. Consequently, from Theorem 1 we deduce that $T$ has at least a fixed point.

Remark 2. The Theorem 4 is followed if $O(f ; t)=t$ and $f=I$ in Theorem 5.

The following Corollary is immediate of Theorem 5.

Corollary 1. Let $C$ be a nonempty, bounded, closed and convex subset of a Banach space $E, T: C \rightarrow C$ and $\psi: R_{+} \rightarrow R_{+}$are continuous functions. Suppose that there exists a constant $0<\lambda<1$ such that for all $X \subseteq C$,

$$
\psi[O(f ; \mu(T X))] \leq \lambda[\psi(O(f ; \mu(X)))] \psi(\mu(X),
$$

where $\mu$ is an arbitrary measure of noncompactness and $O(\bullet ; \cdot) \in \Theta$. Then $T$ has at least one fixed point in $C$. 
Remark 3. The Darbo's fixed point theorem is followed if $O(f ; t)=t, f=I$ and $\psi=I$ in Corollary 1 .

Theorem 6. Let $C$ be a nonempty, bounded, closed and convex subset of a Banach space $E$ and $T: C \times C \times C \rightarrow C$ be a continuous function such that

$$
\begin{array}{r}
\psi\left[O\left(f ; \mu\left(T\left(x_{1} \times x_{2} \times x_{3}\right)\right)\right)\right] \leq \frac{1}{3} \Phi\left[\psi\left(O\left(f ; \mu\left(x_{1}\right)+\mu\left(x_{2}\right)+\mu\left(x_{3}\right)\right)\right]\right. \\
\times \psi\left[\mu\left(x_{1}\right)+\mu\left(x_{2}\right)+\mu\left(x_{3}\right)\right]
\end{array}
$$

for any subset $x_{1}, x_{2}, x_{3}$ of $C$, where $\mu$ is an arbitrary $M N C, \Phi:[0, \infty) \rightarrow[0,1)$ is nondecreasing function such that $\lim _{n \rightarrow \infty} \Phi^{n}(t)=0$ for $t \geq 0$ and $\psi: R_{+} \rightarrow R_{+}$is a continuous function such that $\psi(t)=0$ if and only if $t=0$. Also, $O(\bullet ; \cdot) \in \Theta$ and $O(f ; t+r+s) \leq O(f ; t)+O(f ; s)+O(f ; r)$ for all $t, s, r \geq 0$. Then $T$ has at least a tripled fixed point.

Proof. From Remark 1, we have $\widetilde{\mu}(x)=\mu\left(x_{1}\right)+\mu\left(x_{2}\right)+\mu\left(x_{3}\right)$. Now we define a mapping $\widetilde{T}: C \times C \times C \rightarrow C \times C \times C$ by

$$
\widetilde{T}(x, y, z)=(T(x, y, z), T(y, x, z), T(z, y, x)) .
$$

It is clear that $\widetilde{T}$ is continuous. We prove that $\widetilde{T}$ satisfies all the conditions of Theorem 5. Let $X \subseteq C \times C \times C$ be any nonempty subset by in (2.2) and $2^{\circ}$ we obtain

$$
\begin{aligned}
& \psi\left[O(f ; \widetilde{\mu}(\widetilde{T}(x))] \leq \psi\left[O \left(f ; \widetilde{\mu}\left(T\left(x_{1} \times x_{2} \times x_{3}\right)\right) \times T\left(x_{2} \times x_{1} \times x_{3}\right)\right.\right.\right. \\
&\left.\times T\left(x_{3} \times x_{2} \times x_{1}\right)\right] \\
&= \psi\left[O \left(f ; \mu\left(T\left(x_{1} \times x_{2} \times x_{3}\right)+\mu\left(T\left(x_{2} \times x_{1} \times x_{3}\right)\right.\right.\right.\right. \\
&+ \mu\left(T\left(x_{3} \times x_{2} \times x_{1}\right)\right] \\
&= \psi\left[O\left(f ; \mu\left(T\left(x_{1} \times x_{2} \times x_{3}\right)\right)\right)\right]+\psi\left[O\left(f ; \mu\left(T\left(x_{2} \times x_{1} \times x_{3}\right)\right)\right)\right] \\
&+\psi\left[O\left(f ; \mu\left(T\left(x_{3} \times x_{2} \times x_{1}\right)\right)\right)\right] \\
& \leq \frac{1}{3} \Phi\left[\psi\left(O\left(f ; \mu\left(x_{1}\right)+\mu\left(x_{2}\right)+\mu\left(x_{3}\right)\right)\right] \psi\left[\mu\left(x_{1}\right)+\mu\left(x_{2}\right)+\mu\left(x_{3}\right)\right]\right. \\
&+\frac{1}{3} \Phi\left[\psi\left(O\left(f ; \mu\left(x_{2}\right)+\mu\left(x_{1}\right)+\mu\left(x_{3}\right)\right)\right] \psi\left[\mu\left(x_{2}\right)+\mu\left(x_{1}\right)+\mu\left(x_{3}\right)\right]\right. \\
&+\frac{1}{3} \Phi\left[\psi\left(O\left(f ; \mu\left(x_{3}\right)+\mu\left(x_{2}\right)+\mu\left(x_{1}\right)\right)\right] \psi\left[\mu\left(x_{3}\right)+\mu\left(x_{2}\right)+\mu\left(x_{1}\right)\right]\right. \\
&= \Phi\left[\psi\left(O\left(f ; \mu\left(x_{1}\right)+\mu\left(x_{2}\right)+\mu\left(x_{3}\right)\right)\right] \psi\left[\mu\left(x_{1}\right)+\mu\left(x_{2}\right)+\mu\left(x_{3}\right)\right] .\right.
\end{aligned}
$$

From Theorem 5 deduce that $\widetilde{T}$ has at least a fixed point in $C \times C \times C$ and $T$ has at least a tripled fixed point.

As application of Theorem 6 we can get the following corollary. 
Corollary 2. Let $C$ be a nonempty, bounded, closed and convex subset of a Banach space $E$ and $T: C \times C \times C \rightarrow C$ be a continuous function such that

$$
\mu\left(T\left(x_{1} \times x_{2} \times x_{3}\right)\right) \leq \frac{1}{3} \Phi\left(\mu\left(x_{1}\right)+\mu\left(x_{2}\right)+\mu\left(x_{3}\right)\right),
$$

for any subset $x_{1}, x_{2}, x_{3}$ of $C$, where $\mu$ is an arbitrary MNC. Also, $\Phi:[0, \infty) \rightarrow[0,1)$ is a nondecreasing function such that $\lim _{n \rightarrow \infty} \Phi^{n}(t)=0$ for $t \geq 0$. Then $T$ has at least a tripled fixed point.

\section{APPLICATION}

In this section as an application of Theorem 6 and Corollary 2 we study the existence of solutions for the system of equations (1.1). Let the space $C[0, T]$ consisting of all real functions defined, bounded and continuous on the interval $[0, T]$ equipped with the standard norm

$$
\|x\|=\sup \{|x(t)|: t \in[0, T]\} .
$$

Recall that the modulus of continuity of a function $x \in C[0, T]$ is defined by

$$
\omega(x, \epsilon)=\sup \{|x(t)-x(s)|: t, s \in[0, T],|t-s| \leq \epsilon\} .
$$

Since $x$ is uniformly continuous on $[0, T]$ then $\omega(x, \epsilon) \rightarrow 0$ as $\epsilon \rightarrow 0$ and the Hausdorff measure of noncompactness for all bounded sets $\Omega$ of $C[0, T]$ is equivalent to

See more detail [3].

$$
\chi(\Omega)=\lim _{\epsilon \rightarrow 0}\left\{\sup _{x \in X} \omega(x, \epsilon)\right\} .
$$

Now we consider the following assumptions:

$\left(\mathrm{A}_{1}\right) \zeta, \beta:[0, T] \rightarrow[0, T]$ are continuous functions.

$\left(\mathrm{A}_{2}\right)$ The function $f:[0, T] \times \mathbb{R}^{6} \rightarrow \mathbb{R}$ is continuous and there exist continuous and nondecreasing functions $\varphi, \theta: \mathbb{R}_{+} \longrightarrow \mathbb{R}_{+}$such that $\lim _{n \rightarrow \infty} \Phi^{n}(t)=0$ for $t \geq 0$ and

$$
\begin{array}{r}
\left|f\left(t, x_{1}, x_{2}, \ldots, x_{6}\right)-f\left(t, y_{1}, y_{2}, \ldots, y_{6}\right)\right| \leq \theta\left(\max _{1 \leq i \leq 3}\left|x_{i}-y_{i}\right|\right)+ \\
\frac{1}{3} \varphi\left(\left|x_{4}-y_{4}\right|+\left|x_{5}-y_{5}\right|+\left|x_{6}-y_{6}\right|\right)
\end{array}
$$

$\left(\mathrm{A}_{3}\right)$ There exists a positive solution $r_{0}$ of the inequality

$$
M+\theta(\operatorname{Tr})+\frac{1}{3} \varphi(3 r) \leq r
$$

where

$$
M=\sup \left\{\left|f\left(t, x_{0}, y_{0}, z_{0}, 0,0,0\right)\right|: t \in[0, T]\right\} .
$$


Now, let us put $x^{\prime}(t)=X(t), y^{\prime}(t)=Y(t)$ and $z^{\prime}(t)=Z(t)$. Then the problem (1.1) can be replaced equivalently by the following functional-integral equation

$$
\left\{\begin{array}{rl}
X(t)=f & t, x_{0}+\int_{0}^{\zeta(t)} X(s) d s, y_{0} \\
& +\int_{0}^{\zeta(t)} Y(s) d s, z_{0}+\int_{0}^{\zeta(t)} Z(s) d s, X(\beta(t), Y(\beta(t), Z(\beta(t)) \\
Y(t)=f & \left(t, y_{0}+\int_{0}^{\zeta(t)} Y(s) d s, x_{0}\right. \\
& +\int_{0}^{\zeta(t)} X(s) d s, z_{0}+\int_{0}^{\zeta(t)} Z(s) d s, Y(\beta(t), X(\beta(t), Z(\beta(t)) \\
Z(t)=f & \left(t, z_{0}+\int_{0}^{\zeta(t)} Z(s) d s, y_{0}\right. \\
& +\int_{0}^{\zeta(t)} Y(s) d s, x_{0}+\int_{0}^{\zeta(t)} X(s) d s, X(\beta(t), Y(\beta(t), Z(\beta(t))
\end{array}\right.
$$

where $t \in[0, T]$. In the sequel we will examine the equation (3.3).

Theorem 7. Under the assumptions $\left(A_{1}\right)-\left(A_{3}\right)$ the equation (3.3) has at least one solution $x \in\{C[0, T]\}^{3}$

Proof. Let us consider the operator $T$ defined on the space $C[0, T]$ by the formula

$$
\begin{aligned}
T(x, y, z)(t)= & f\left(t, x_{0}+\int_{0}^{\zeta(t)} X(s) d s, y_{0}+\int_{0}^{\zeta(t)} Y(s) d s, z_{0}\right. \\
& +\int_{0}^{\zeta(t)} Z(s) d s, X(\beta(t), Y(\beta(t), Z(\beta(t))
\end{aligned}
$$

for all $t \in[0, T]$. Observe that in view of assumptions $\left(A_{1}\right)$ and $\left(A_{2}\right)$, the function $T x$ is continuous on $[0, T]$ for any $x \in C[0, T]$, i.e. $T$ transforms the space $C[0, T]$ into itself. For arbitrarily fixed $t \in[0, T]$, we have

$$
\begin{aligned}
|T(x, y, z)(t)| \leq & f\left(t, x_{0}+\int_{0}^{\zeta(t)} x(s) d s, y_{0}+\int_{0}^{\zeta(t)} y(s) d s, z_{0}\right. \\
& \left.+\int_{0}^{\zeta(t)} z(s) d s, x(\beta(t)), y(\beta(t)), z(\beta(t))\right) \\
& -f\left(t, x_{0}, y_{0}, z_{0}, 0,0,0\right)|+| f\left(t, x_{0}, y_{0}, z_{0}, 0,0,0\right) \mid \\
\leq & \theta\left(\max \left\{\left|\int_{0}^{\zeta(t)} x(s) d s\right|,\left|\int_{0}^{\zeta(t)} y(s) d s\right|,\left|\int_{0}^{\zeta(t)} z(s) d s\right|\right\}\right) \\
& +\frac{1}{3} \varphi(|x(\beta(t))|+|y(\beta(t))|+|z(\beta(t))|) \\
& +\left|f\left(t, x_{0}, y_{0}, z_{0}, 0,0,0\right)\right| \\
\leq & \theta(T \max \{\|x\|,\|y\|,\|z\|\})+\frac{1}{3} \varphi(\|x\|+\|y\|+\|z\|)+M
\end{aligned}
$$


Thus,

$$
\|T(x, y, z)\| \leq \theta(T \max \{\|x\|,\|y\|,\|z\|\})+\frac{1}{3} \varphi(\|x\|+\|y\|+\|z\|)+M
$$

and $T(x, y, z) \in C[0, T]$ for any $(x, y, z) \in(C[0, T])^{3}$. Due to Inequality (3.2) the function $T$ maps $\left(\bar{B}_{r_{0}}\right)^{3}$ into $\bar{B}_{r_{0}}$. Now we show that $T$ is a continuous function on $\left(\bar{B}_{r_{0}}\right)^{3}$. To do this, let us fix $\varepsilon>0$ and take arbitrary $(x, y, z),(u, v, w) \in\left(\bar{B}_{r_{0}}\right)^{3}$ such that $\max \{\|x-u\|,\|y-v\|,\|z-w\|\}<\varepsilon$. Then, for $t \in[0, T]$, we have

$$
\begin{aligned}
|T(x, y, z)(t)-T(u, v, w)(t)| \leq & \mid f\left(t, x_{0}+\int_{0}^{\zeta(t)} x(s) d s, y_{0}+\int_{0}^{\zeta(t)} y(s) d s, z_{0}\right. \\
& \left.+\int_{0}^{\zeta(t)} z(s) d s, x(\beta(t)), y(\beta(t)), z(\beta(t))\right) \\
& -f\left(t, x_{0}+\int_{0}^{\zeta(t)} u(s) d s, y_{0}+\int_{0}^{\zeta(t)} v(s) d s, z_{0}\right. \\
& \left.+\int_{0}^{\zeta(t)} w(s) d s, u(\beta(t)), v(\beta(t)), w(\beta(t))\right) \mid \\
\leq & \theta\left(\operatorname { m a x } \left\{\int_{0}^{\zeta(t)}|x(s)-u(s)| d s, \int_{0}^{\zeta(t)}|y(s)-v(s)| d s,\right.\right. \\
& \left.\left.\int_{0}^{\zeta(t)}|z(s)-w(s)| d s\right\}\right) \\
& +\frac{1}{3} \varphi(|x(\beta(t))-u(\beta(t))|+|y(\beta(t))-v(\beta(t))| \\
& +|z(\beta(t))-w(\beta(t))|) \\
\leq & \theta(T \varepsilon)+\frac{1}{3} \varphi(3 \varepsilon)
\end{aligned}
$$

So we have $\theta(T \varepsilon)+\frac{1}{3} \varphi(3 \varepsilon) \longrightarrow 0$ as $\varepsilon \longrightarrow 0$. Thus $T$ is a continuous function on $\left(\bar{B}_{r_{0}}\right)^{3}$. Now we show that $T$ satisfies all the conditions of Corollary 2. Let $X_{1}, X_{2}$ and $X_{3}$ be nonempty and bounded subsets of $\bar{B}_{r_{0}}$. Assume that $\varepsilon>0$ is arbitrary constant. Also we take $t_{1}, t_{2} \in[0, T]$, with $\left|t_{2}-t_{1}\right| \leq \varepsilon$ and $x \in X_{1}, y \in X_{2}$ and $z \in X_{3}$. Then we have 


$$
\begin{aligned}
& \left|T(x, y, z)\left(t_{1}\right)-T(x, y, z)\left(t_{2}\right)\right| \leq \mid f\left(t_{1}, x_{0}+\int_{0}^{\zeta\left(t_{1}\right)} x(s) d s, y_{0}+\int_{0}^{\zeta\left(t_{1}\right)} y(s) d s, z_{0}\right. \\
& \left.+\int_{0}^{\zeta\left(t_{1}\right)} z(s) d s, x\left(\beta\left(t_{1}\right)\right), y\left(\beta\left(t_{1}\right)\right), z\left(\beta\left(t_{1}\right)\right)\right) \\
& -f\left(t_{1}, x_{0}+\int_{0}^{\zeta\left(t_{1}\right)} x(s) d s, y_{0}+\int_{0}^{\zeta\left(t_{1}\right)} y(s) d s, z_{0}\right. \\
& \left.+\int_{0}^{\zeta\left(t_{1}\right)} z(s) d s, x\left(\beta\left(t_{2}\right)\right), y\left(\beta\left(t_{2}\right)\right), z\left(\beta\left(t_{2}\right)\right)\right) \mid \\
& +\mid f\left(t_{1}, x_{0}+\int_{0}^{\zeta\left(t_{1}\right)} x(s) d s, y_{0}+\int_{0}^{\zeta\left(t_{1}\right)} y(s) d s, z_{0}\right. \\
& \left.+\int_{0}^{\zeta\left(t_{1}\right)} z(s) d s, x\left(\beta\left(t_{2}\right)\right), y\left(\beta\left(t_{2}\right)\right), z\left(\beta\left(t_{2}\right)\right)\right) \\
& -f\left(t_{2}, x_{0}+\int_{0}^{\zeta\left(t_{1}\right)} x(s) d s, y_{0}+\int_{0}^{\zeta\left(t_{1}\right)} y(s) d s, z_{0}\right. \\
& \left.+\int_{0}^{\zeta\left(t_{1}\right)} z(s) d s, x\left(\beta\left(t_{2}\right)\right), y\left(\beta\left(t_{2}\right)\right), z\left(\beta\left(t_{2}\right)\right)\right) \mid \\
& +\mid f\left(t_{2}, x_{0}+\int_{0}^{\zeta\left(t_{1}\right)} x(s) d s, y_{0}+\int_{0}^{\zeta\left(t_{1}\right)} y(s) d s, z_{0}\right. \\
& \left.+\int_{0}^{\zeta\left(t_{1}\right)} z(s) d s, x\left(\beta\left(t_{2}\right)\right), y\left(\beta\left(t_{2}\right)\right), z\left(\beta\left(t_{2}\right)\right)\right) \\
& -f\left(t_{2}, x_{0}+\int_{0}^{\zeta\left(t_{1}\right)} x(s) d s, y_{0}+\int_{0}^{\zeta\left(t_{1}\right)} y(s) d s, z_{0}\right. \\
& \left.+\int_{0}^{\zeta\left(t_{1}\right)} z(s) d s, x\left(\beta\left(t_{2}\right)\right), y\left(\beta\left(t_{2}\right)\right), z\left(\beta\left(t_{2}\right)\right)\right) \mid \\
& +\mid f\left(t_{2}, x_{0}+\int_{0}^{\zeta\left(t_{1}\right)} x(s) d s, y_{0}+\int_{0}^{\zeta\left(t_{1}\right)} y(s) d s, z_{0}\right. \\
& \left.+\int_{0}^{\zeta\left(t_{1}\right)} z(s) d s, x\left(\beta\left(t_{2}\right)\right), y\left(\beta\left(t_{2}\right)\right), z\left(\beta\left(t_{2}\right)\right)\right) \\
& -f\left(t_{2}, x_{0}+\int_{0}^{\zeta\left(t_{1}\right)} x(s) d s, y_{0}+\int_{0}^{\zeta\left(t_{1}\right)} y(s) d s, z_{0}\right. \\
& \left.+\int_{0}^{\zeta\left(t_{1}\right)} z(s) d s, x\left(\beta\left(t_{2}\right)\right), y\left(\beta\left(t_{2}\right)\right), z\left(\beta\left(t_{2}\right)\right)\right) \mid \\
& \leq \frac{1}{3} \varphi\left(\left|x\left(\beta\left(t_{1}\right)\right)-x\left(\beta\left(t_{2}\right)\right)\right|+\left|y\left(\beta\left(t_{1}\right)\right)-y\left(\beta\left(t_{2}\right)\right)\right|\right. \\
& \left.+\left|z\left(\beta\left(t_{1}\right)\right)-z\left(\beta\left(t_{2}\right)\right)\right|\right)+\omega(f, \varepsilon) \\
& +\theta\left(\max \left\{\int_{\zeta\left(t_{2}\right)}^{\zeta\left(t_{1}\right)}|x(s)| d s, \int_{\zeta\left(t_{2}\right)}^{\zeta\left(t_{1}\right)} \mid y(s)\right) \mid d s,\right. \\
& \left.\left.\int_{\zeta\left(t_{2}\right)}^{\zeta\left(t_{1}\right)}|z(s)| d s\right\}\right) \\
& \leq \frac{1}{3} \varphi(\omega(x, \omega(\beta, \varepsilon))+\omega(y, \omega(\beta, \varepsilon))+\omega(z, \omega(\beta, \varepsilon))) \\
& +\omega(f, \varepsilon)+\theta\left(\max \left\{r_{0} \omega(\zeta, \varepsilon)\right\}\right),
\end{aligned}
$$


where

$$
\begin{aligned}
& \omega(\beta, \varepsilon)=\sup \left\{\left|\beta\left(t_{2}\right)-\beta\left(t_{1}\right)\right|:\left|t_{1}-t_{2}\right| \leq \varepsilon, t_{1}, t_{2} \in[0, T]\right\} \\
& \omega(\zeta, \varepsilon)=\sup \left\{\left|\zeta\left(t_{2}\right)-\zeta\left(t_{1}\right)\right|: t_{1}, t_{2} \in[0, T],\left|t_{1}-t_{2}\right| \leq \varepsilon\right\} \\
& \omega(x, \omega(\beta, \varepsilon))=\sup \left\{\left|x\left(t_{2}\right)-x\left(t_{1}\right)\right|:\left|t_{1}-t_{2}\right| \leq \omega(\beta, \varepsilon), t_{1}, t_{2} \in[0, \beta(T)]\right\}, \\
& \omega(f, \varepsilon)=\sup \left\{\left|f\left(t_{1}, x_{1}, x_{2}, \ldots x_{6}\right)-f\left(t_{2}, x_{1}, x_{2}, \ldots x_{6}\right)\right|:\left|t_{1}-t_{2}\right| \leq \varepsilon,\right. \\
& \left.t_{1}, t_{2} \in[0, T], x_{4}, x_{5}, x_{6} \in\left[-r_{0}, r_{0}\right], x_{1}, x_{2}, x_{3} \in\left[-\beta(T) r_{0}, \beta(T) r_{0}\right]\right\} .
\end{aligned}
$$

We obtained

$$
\begin{aligned}
\left|T(x, y, z)\left(t_{1}\right)-T(x, y, z)\left(t_{2}\right)\right| \leq & \frac{1}{3} \varphi(\omega(x, \omega(\beta, \varepsilon))+\omega(y, \omega(\beta, \varepsilon))+\omega(z, \omega(\beta, \varepsilon))) \\
& +\omega(f, \varepsilon)+\theta\left(\max \left\{r_{0} \omega(\zeta, \varepsilon)\right\}\right)
\end{aligned}
$$

Therefore, we have

$$
\begin{aligned}
\omega\left(T\left(X_{1} \times X_{2} \times X_{3}\right), \varepsilon\right) \leq & \frac{1}{3} \varphi\left(\omega\left(X_{1}, \omega(\beta, \varepsilon)\right)+\omega\left(X_{2}, \omega(\beta, \varepsilon)\right)+\omega\left(X_{3}, \omega(\beta, \varepsilon)\right)\right) \\
& +\omega(f, \varepsilon))+\theta\left(\max \left\{r_{0} \omega(\zeta, \varepsilon)\right\} .\right.
\end{aligned}
$$

On the other hand by the uniform continuity of $f, \beta$ and $\zeta$ on $[0, T] \times\left[-\beta(T) r_{0}, \beta(T) r_{0}\right]^{3} \times$ $\left[-r_{0}, r_{0}\right]^{3},[0, T]$ and $[0, T]$ respectively, we have $\omega(f, \varepsilon) \longrightarrow 0, \omega(\beta, \varepsilon)$ and $\omega(\zeta, \varepsilon) \longrightarrow 0$ as $\varepsilon \longrightarrow 0$. Therefore we obtain

$$
\mu\left(T\left(X_{1} \times X_{2} \times X_{3}\right) \leq \frac{1}{3} \varphi\left(\mu\left(X_{1}\right)+\mu\left(X_{2}\right)+\mu\left(X_{3}\right)\right) .\right.
$$

By applying Corollary 2, $T$ has a fixed point.

Example 2. Consider the following system of functional differential equations

$$
\left\{\begin{array}{l}
x^{\prime}(t)=t^{2}+\sqrt[3]{x(t)}+\sqrt[5]{y(t)}+\sqrt[7]{z(t)}+\frac{1}{3} \ln \left(1+\left|x^{\prime}(t)+y^{\prime}(t)+z^{\prime}(t)\right|\right) \\
y^{\prime}(t)=t^{2}+\sqrt[3]{y(t)}+\sqrt[5]{x(t)}+\sqrt[7]{z(t)}+\frac{1}{3} \ln \left(1+\left|x^{\prime}(t)+y^{\prime}(t)+z^{\prime}(t)\right|\right) \\
z^{\prime}(t)=t^{2}+\sqrt[3]{z(t)}+\sqrt[5]{y(t)}+\sqrt[7]{x(t)}+\frac{1}{3} \ln \left(1+\left|x^{\prime}(t)+y^{\prime}(t)+z^{\prime}(t)\right|\right)
\end{array}\right.
$$

where $t \in[0,3]$ with the initial condition

$$
x(0)=3, y(0)=1, z(0)=2 .
$$

Eq. (3.6) is a special case of Eq. (1.1) where

$$
\beta(t)=\zeta(t)=t
$$




$$
f\left(t, x_{1}, \ldots, x_{6}\right)=t^{2}+\sqrt[3]{x_{1}}+\sqrt[5]{x_{2}}+\sqrt[7]{x_{3}}+\frac{1}{3} \ln \left(1+\left|x_{4}+x_{5}+x_{6}\right|\right) .
$$

From the definitions of $\beta$ and $\zeta$, hypothesis $\left(\mathrm{A}_{1}\right)$ are obviously satisfied. Suppose that $t \in[0,3]$. Now, by taking $\varphi(t)=\ln (1+t), \theta(t)=\max _{i=3,5,7}\{\sqrt[i]{t}\}$ we have

$$
\begin{aligned}
&\left|f\left(t, x_{1}, \ldots, x_{6}\right)-f\left(t, y_{1}, \ldots, y_{6}\right)\right| \leq\left|\sqrt[3]{x_{1}}-\sqrt[3]{y_{1}}\right|+\left|\sqrt[5]{x_{2}}-\sqrt[5]{y_{2}}\right|+\left|\sqrt[7]{x_{3}}-\sqrt[7]{y_{3}}\right| \\
&+\frac{1}{3} \mid \ln \left(1+\left|x_{4}+x_{5}+x_{6}\right|\right) \\
& \quad-\ln \left(1+\left|y_{4}+y_{5}+y_{6}\right|\right) \\
& \leq\left|\sqrt[3]{x_{1}}-\sqrt[3]{y_{1}}\right|+\left|\sqrt[5]{x_{2}}-\sqrt[5]{y_{2}}\right|+\left|\sqrt[7]{x_{3}}-\sqrt[7]{y_{3}}\right| \\
&+\frac{1}{3} \ln \left(1+\frac{\left|x_{4}+x_{5}+x_{6}\right|-\left|y_{4}+y_{5}+y_{6}\right|}{1+\left|y_{4}+y_{5}+y_{6}\right|}\right) \\
& \leq \sqrt[3]{\left|x_{1}-y_{1}\right|}+\sqrt[5]{\left|x_{2}-y_{2}\right|}+\sqrt[7]{\left|x_{3}-y_{3}\right|} \\
&+\frac{1}{3} \ln \left(1+\left|x_{4}+x_{5}+x_{6}-\left(y_{4}+y_{5}+y_{6}\right)\right|\right) \\
& \leq \sqrt[3]{\left|x_{1}-y_{1}\right|}+\sqrt[5]{\left|x_{2}-y_{2}\right|}+\sqrt[7]{\left|x_{3}-y_{3}\right|} \\
&+\frac{1}{3} \ln \left(1+\left|x_{4}-y_{4}\right|+\left|x_{5}-y_{5}\right|+\left|x_{6}-y_{6}\right|\right) \\
&= \frac{1}{3} \varphi\left(\left|x_{4}-y_{4}\right|+\left|x_{5}-y_{5}\right|+\left|x_{6}-y_{6}\right|\right) \\
&+\theta\left(\max _{i=1,2,3}\left\{\left|x_{i}-y_{i}\right|\right\}\right) .
\end{aligned}
$$

Thus, from (3.7) we infer that condition $\left(\mathrm{A}_{2}\right)$ holds. Furthermore,

$$
\begin{aligned}
M & =\sup \left\{\left|f\left(t, x_{0}, y_{0}, z_{0}, 0,0,0\right)\right|: t \in[0, T]\right\} \\
& =\sup \left\{t^{2}+\sqrt[3]{3}+\sqrt[5]{1}+\sqrt[7]{2}: t \in[0,3]\right\} \\
& \leq 12 .
\end{aligned}
$$

It is easy to see that each number $r \geq 20$ satisfies the inequality in condition $\left(\mathrm{A}_{3}\right)$, i.e.,

$$
12+\theta(3 r)+\frac{1}{3} \varphi(3 r) \leq r
$$

Thus, as the number $r_{0}$ we can take $r_{0}=20$. Consequently, all the conditions of Theorem 7 are satisfied. Hence the system equations 3.6 has at least one solution which belong to the space $\{C[0, T]\}^{3}$.

\section{REFERENCES}

[1] A. Aghajani, R. Allahyari, and M. Mursaleen, "A generalization of darbo's theorem with application to the solvability of systems of integral equations." Journal of Computational and Applied Mathematics, vol. 260, no. 2, pp. 68-77, 2014, doi: 10.1016/j.cam.2013.09.039. 
[2] A. Aghajani, J. Banaś , and N. Sabzali, "Some generalizations of Darbo fixed point theorem and applications." Bulletin of the Belgian Mathematical Society, vol. 20, no. 2, pp. 345-358, 2013.

[3] R. R. Akhmerov, M. I. Kamenskii, A. S. Potapov, A. E. Rodkina, and B. N. Sadovskii, Measures of Noncompactness and Condensing Operators. Basel: Birkhäuser, 1992. doi: 10.1007/978-30348-5727-7.

[4] I. Altun and D. Turkoglu, "A fixed point theorem for mappings satisfying a general contractive condition of operator type." Journal of Computational Analysis and Applications, vol. 9, no. 1, pp. 9-14, 2007.

[5] J. Banaś and R. Nalepa, "On a measure of noncompactness in the space of functions with tempered increments." Journal of Mathematical Analysis and Applications, vol. 435, no. 2, pp. 1634-1651, 2016, doi: 10.1016/j.jmaa.2015.11.033.

[6] J. Banas and K. Goebel, Measures of noncompactness in Banach spaces, ser. Lecture Notes in Pure and Applied Mathematics. New York, Basel: Marcel Dekker, Inc., 1980, vol. 60.

[7] G. Darbo, "Punti uniti in trasformazioni a codominio non compatto," Rendiconti del Seminario Matematico della Universita di Padova, vol. 24, pp. 84-92, 1955.

[8] V. Karakaya, N. E. H. Bouzara, and Y. Atalan, "Existence of tripled fixed points for a class of condensing operators in banach spaces," The Scientific World Journal, 2014, doi: $10.1155 / 2014 / 541862$.

[9] C. Kuratowski, "Sur les espaces complets," Fundamenta Mathematicae, vol. 15, no. 1, pp. 301309, 1930.

[10] A. Samadi and M. B. Ghaemi, " An extension of Darbo fixed point theorem and its applications to coupled fixed point and integral equations.” Filomat, vol. 28, no. 4, pp. 879-886, 2014, doi: 10.2298/FIL1404879S.

Authors' addresses

Sh. Banaei

Department of Mathematics, Karaj Branch, Islamic Azad University, Karaj, Iran

E-mail address: sh.banaei@kiau.ac.ir

M. B. Ghaemi

Department of Mathematics, Karaj Branch, Islamic Azad University, Karaj, Iran

E-mail address: mghaemi@iust.ac.ir

R. Saadati

Department of Mathematics, Iran University of Science and Technology, Iran

E-mail address: rsaadati@iust.ac.ir 